\title{
Carry-free models and beyond
}

\author{
Se Yong Park, Gireeja Ranade and Anant Sahai \\ Wireless Foundations, EECS, UC Berkeley \\ \{separk, gireeja, sahai\}@eecs.berkeley.edu
}

\begin{abstract}
The generalized deterministic models recently proposed by Niesen and Maddah-Ali [1] successfully capture realinterference alignment as observed in Gaussian models. Simpler deterministic models, like ADT models [2], cannot demonstrate this phenomenon because they are limited in the set of channel gains they can model. This paper reinterprets the Niesen and Maddah-Ali models through the lens of carry-free operations. We further explore these carry-free models by considering i.i.d. unknown fading networks. In the unknown fading context, a carry-free model can be further simplified to a maxsuperposition model, where signals are superposed by a nonlinear max operation. Unlike in relay-networks with known fading and linear superposition, we find that decode-and-forward can perform arbitrarily better than compress-and-forward in maxsuperposition relay networks with unknown fading.
\end{abstract}

\section{INTRODUCTION}

Channel models have always formed the foundation for understanding and engineering wireless communication systems. The choice of model involves a tradeoff between simplicity and "distance" from the real-world. Fig. 1 shows a spectrum of these models. Rayleigh-faded Gaussian models are among the most commonly used and have undoubtedly furthered the state-of-the-art in the field. However, these models are complex, and this complexity can obscure the essential interaction between channels and the information flowing through them.

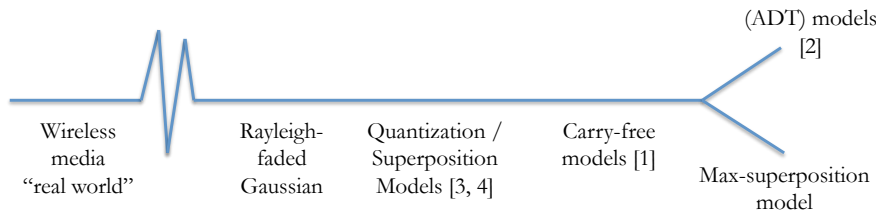

Fig. 1. Models for wireless media. Carry-free models lie between the Rayleigh-faded Gaussian and quantization models on one side, and ADT models on the other.

This led to the development of the simple deterministic "bubble-model" by Avestimehr, Diggavi and Tse (ADT model) [2], which pictorially represents information flows. The simplicity of these models led to progress on the relay and interference channels. However, the same simplicity prevents them from capturing the interference alignment phenomena observed in Gaussian fading channels. ADT models only model gains that are powers of two-they are limited in their ability to capture real-valued channel gains.

Real interference alignment exploits channel diversity and channel state information at the transmitter. Channel gains that
TABLE I

A COMPARISON OF COMMUNICATION MODELS

\begin{tabular}{|c|c|c|c|c|}
\hline & $\begin{array}{c}\text { Faded } \\
\text { Gaussian }\end{array}$ & $\begin{array}{c}\text { Quantization } \\
\text { [3], [4] }\end{array}$ & $\begin{array}{c}\text { Carry-free } \\
\text { [1] }\end{array}$ & $\begin{array}{c}\text { ADT } \\
\text { [2] }\end{array}$ \\
\hline \hline $2 \times 2$ IC & $\checkmark$ & $\checkmark$ & $\checkmark$ & $\checkmark$ \\
\hline Relay channel & $\checkmark$ & $\checkmark$ & $\checkmark$ & $\checkmark$ \\
\hline $\begin{array}{c}\left(\frac{1}{2}, \frac{1}{2}\right) \text { d.o.f. } \\
\text { in MAC w/o } \\
\text { timesharing \& CSIT }\end{array}$ & $\checkmark$ & $\checkmark$ & $\checkmark$ & $\times$ \\
\hline Real IA & $\checkmark$ & $\checkmark$ & $\checkmark$ & $\times$ \\
\hline $\begin{array}{c}\text { Direct map to } \\
\text { Gaussian Networks }\end{array}$ & $\checkmark$ & $\checkmark$ & $\times$ & $\times$ \\
\hline Beamforming gain & $\checkmark$ & $\checkmark$ & $\times$ & $\times$ \\
\hline Unknown fading & $\checkmark$ & $\checkmark$ & $\checkmark$ & $\times$ \\
\hline
\end{tabular}

are not just powers of two are essential to capture it. However, the naive approach leads to a different problem: the "carries" in the multiplication of the channel gain and the signal break the bit-wise linearity (and hence the simplicity) of the model. To circumvent this issue, the models recently proposed by Maddah-Ali and Niesen [1] ignore these carries to preserve linearity and yet model generic channel gains. This inspires us to call these models "carry-free" models. Essentially, these carry-free models replace a single real channel input by a discrete valued signal and a real channel gain by an LTI system (Fig. 2). Sections II and III give details of the carry-free interpretation of [1]. Section IV deals with MIMO channels.

As a summary, Table I provides a comparison of the different families of models. While neither ADT nor carryfree models provide direct mappings for individual instances of Gaussian networks, carry-free models are able to capture fractional degrees of freedom and real interference alignment.

Armed with this new richer model, Section V attempts to understand another Gaussian phenomenon: communication over unknown i.i.d fading channels. A first curiosity about such channels was conjectured in 1969 [5] - even for continuous fading distributions the optimal input distribution is discrete. Since then many works have followed up to characterize unknown point-to-point models [6], [7], [8]. Gaussian channel capacities in this case behave as $\log (\log S N R)$, as opposed to the $\log S N R$ scaling for channels with known fading.

However, carry-free models for unknown fading channels are not deterministic. The input-output relationship in these models still contains the randomness from the unknown channel fading. We propose the max-superposition model in Section V that removes this randomness. The model builds from the observation that only the highest non-zero bit in a signal conveys information when the channel is unknown. The max-model explains how the energy level modulation 
scheme, which is optimal in the case of real channels [8], can in fact be thought of as simple pulse-position-modulation. A similar fact is true in networks with unknown fading. In the max-superposition model, superimposed signals are combined through the non-linear max-operator, as opposed to a linear sum (hence, the name).

Finally, we note that the max-superposition model of the relay channel offers a surprise: partial-decode-and-forward can offer an unbounded gain over compress-and-forward.

\section{CARRY-FREE OPERATIONS}

Just as in [2], the generalized deterministic models of [1] consider the binary-string representation of real numbers as a starting point. To interpret these models as "carry-free" models we define carry-free arithmetic. Consider such a string $a_{n} a_{n-1} \ldots a_{1} a_{0} a_{-1} \ldots$. Let $\mathbb{B}$ be the set of all binary strings of infinite length. We define carry-free addition, $\oplus_{c}$, and multiplication, $\otimes_{c}$, over $\mathbb{B}$ in the obvious way.

\section{A. Addition and Multiplication}

Let $c_{n} \ldots c_{1} c_{0} c_{-1} \ldots=a_{n} \ldots a_{1} a_{0} a_{-1} \ldots \oplus_{c} b_{n} \ldots b_{1} b_{0} b_{-1} \ldots$ Then $c_{i}=a_{i}+b_{i}$, where the sum is over $\mathbb{F}_{2}$. The addition operation involves no carryovers unlike in real addition. We derive the name "carry-free" from this property. Bit interactions at one level do not affect higher level bits.

Let $c_{n} \ldots c_{1} c_{0} c_{-1} \ldots=a_{n} \ldots a_{1} a_{0} a_{-1} \ldots \otimes_{c} b_{n} \ldots b_{1} b_{0} b_{-1} \ldots$. Then $c_{i}=\sum_{k} a_{k} b_{i-k}$, where the summations are in $\mathbb{F}_{2}$. Thus, multiplication is basically "convolution" (Fig. 2), as noted in [1]. We note that it is commutative and associative.

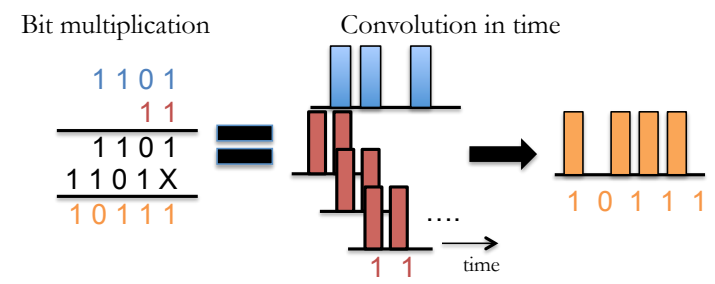

Fig. 2. Carry-free multiplication as "convolution"

\section{B. Signal notation}

The bit levels of carry-free numbers are like a fake "time". Signals convolve to interact-just as carry-free strings multiply. The string $a_{n} \ldots a_{1} a_{0} a_{-1} \ldots$ can also be denoted as signal samples from $-\infty$ to $n$ : $a[n] a[n-1] \ldots a[0] a[-1] \ldots$

\section{Polynomial notation}

Under the multiplication and addition defined above, $\left(\mathbb{B}, \oplus_{c}, \otimes_{c}\right)$ forms a commutative ring. There is a natural mapping from this ring to the polynomial ring. $a_{n} \ldots a_{1} a_{0} a_{-1} \ldots$ in $\mathbb{B}$ corresponds to $a_{n} z^{n}+\ldots+a_{1} z+a_{0}+a_{-1} z^{-1} \ldots$. We can check the natural addition and multiplication in the polynomial ring is equivalent to $\oplus_{c}$ and $\otimes_{c}$ of $\mathbb{B}$.

\section{Truncation}

We will use a truncation operation similar to [2], [3], [4] to capture the effect of noise in a channel. For a polynomial $a(z)=\sum_{i} a_{i} z^{i}$, we define $\lfloor a(z)\rfloor=\sum_{i \geq 0} a_{i} z^{i}$.

\section{CARRY-FREE COMMUNICATION MODELS}

\section{A. The point-to-point carry-free model}

Consider a real Gaussian point-to-point channel, $y=$ $2^{n} h x+w . h$ is a fixed realization of a random channel gain uniform in $[0,1)$. The noise $w$ is $\mathcal{N}(0,1)$. For simplicity, we assume signal $x$ has a peak-power constraint of 1 as in [2], and $\left(2^{n}\right)^{2}$ represents the signal-to-noise ratio $(S N R)$. We develop a carry-free model for this channel, parallel to the development of the ADT model, and consider bounded noise.

Considering the binary expansions of $h$ and $x$, we have $y=2^{n}\left(h_{-1} 2^{-1}+h_{-2} 2^{-2}+\ldots\right)\left(x_{-1} 2^{-1}+x_{-2} 2^{-2}+\ldots\right)+$ $\left(w_{-1} 2^{-1}+w_{-2} 2^{-2}+\ldots\right)$. Replacing the binary expansion with polynomial notation, we get $y(z)=z^{n}\left(h_{-1} z^{-1}+h_{-2} z^{-2}+\right.$ $\ldots)\left(x_{-1} z^{-1}+x_{-2} z^{-2}+\ldots\right)+\left(w_{-1} z^{-1}+w_{-2} z^{-2}+\ldots\right)$, which we will shorten as $y(z)=z^{n} h(z) x(z)+w(z)$. We can think of $w_{-i}$ as fair coin flips and write the channel equation as $y(z)=\left\lfloor z^{n} h(z) x(z)\right\rfloor$. Here, $h(z)$ is the channel polynomial, $n=\frac{1}{2} \log (S N R)$ and $x(z)$ is the message polynomial. In matrix form ${ }^{1}$ this is:

$$
\left[\begin{array}{c}
y_{0} \\
y_{1} \\
\vdots \\
y_{n-3} \\
y_{n-2}
\end{array}\right]=\left[\begin{array}{ccccc}
h_{-1} & h_{-2} & \cdots & h_{-(n-2)} & h_{-(n-1)} \\
0 & h_{-1} & \cdots & h_{-(n-3)} & h_{-(n-2)} \\
\vdots & \vdots & \ddots & \vdots & \vdots \\
0 & 0 & \cdots & h_{-1} & h_{-2} \\
0 & 0 & \cdots & 0 & h_{-1}
\end{array}\right]\left[\begin{array}{c}
x_{-(n-1)} \\
x_{-(n-2)} \\
\vdots \\
x_{-2} \\
x_{-1}
\end{array}\right]
$$

or $y=H \cdot x$. Thus, the capacity of the channel is the rank of the $(n-1) \times(n-1)$ matrix $H$, which is determined by the highest location where $h_{i}$ is non-zero.

Theorem 3.1: The capacity of the carry-free point-to-point channel with the channel coefficient $h(z)=\sum_{i} h_{i} z^{i}$ and $\frac{1}{2} \log (\mathrm{SNR})=n$ is $\operatorname{Rank}(H)=n-\min \left\{i \mid h_{-i} \neq 0\right\}$, where $H$ is the matrix as above.

Proof: See Niesen and Maddah-Ali [1].

\section{B. Real-interference alignment in carry-free models}

The crux of real interference alignment lies in understanding fractional degrees of freedom. A minimal model to understand this is the multiple-access channel. An interesting question about fractional degrees of freedom was implicitly posed and answered positively in [9], [10]: can we achieve $\left(\frac{1}{2}, \frac{1}{2}\right)$ d.o.f. without time-sharing or $\mathrm{CSIT}^{2}$ (channel state information at transmitters)? This question is answered for carry-free models by Niesen and Maddah-Ali [1].

\footnotetext{
${ }^{1}$ The triangular structure of this matrix motivates the name lower triangular models in [1].

${ }^{2}$ Successive-decoding schemes in Gaussian models are inherently asymmetric. For instance, consider a scheme where one encoder reduces its power to $\sqrt{P}$ while the other transmits at full power $P$. This requires a pre-coordination on which encoder must reduce power and is equivalent to a time-sharing scheme in cooperation burden. The same must occur in ADT models since the bit shift alignments at the receiver are unknown to the transmitter.
} 


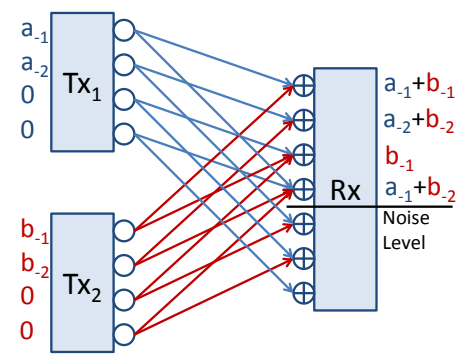

Fig. 3. MAC with channel gains $h(z)=z^{4}\left(1+z^{-3}\right), h^{\prime}(z)=z^{4}(1+$ $\left.z^{-2}\right)$. $\left(\frac{1}{2}, \frac{1}{2}\right)$ d.o.f. are achievable using the scheme described.

Consider a carry-free MAC with no CSIT: $y(z)=$ $\left\lfloor 2^{n} h(z) a(z)+2^{n} h^{\prime}(z) b(z)\right\rfloor$ with $n=\frac{1}{2} \log (S N R)$. In other words, $h(z)$ and $h^{\prime}(z)$ are only known to the receiver, and transmitters are not allowed any cooperation or common randomness for time-sharing. Clearly, the cut-set bound implies that the total d.o.f. of the channel is bounded by 1 . The scheme follows [9], [1], and sets the lower order bits to zero as described in Fig. 3.

Theorem 3.2: Each transmitter can achieve $\frac{1}{2}$ d.o.f. without timesharing or CSIT for almost all channel gains.

Proof: See Niesen and Maddah-Ali [1].

The natural next channel to explore is the X-channel, and here too, carry-free models can capture the notion of fractional degrees of freedom as in the Gaussian case, as shown in [1].

\section{MODELING POINT-TO-POINT MIMO}

The following $2 \times 2$ MIMO example from [2] illustrates a limitation of the ADT model:

$$
Y=2^{k}\left[\begin{array}{ll}
\frac{3}{4} & 1 \\
1 & 1
\end{array}\right] X+N
$$

This channel has 2 d.o.f under the Gaussian model, but only one under ADT models. However, carry-free models represent the channel as $h_{11}(z)=z^{k-1}+z^{k-2}, h_{12}(z)=z^{k}, h_{21}(z)=$ $z^{k}, h_{22}(z)=z^{k}$ and can capture the 2 d.o.f.

Unfortunately, the carry-free model cannot solve this problem generally. There is no simple correspondence between a specific instance of a real $2 \times 2$ MIMO model and a specific carry-free model. Consider the channel

$$
Y=2^{k}\left[\begin{array}{ll}
9 & 3 \\
3 & 1
\end{array}\right] X+N,
$$

with 1 d.o.f. under the real Gaussian model. However, the corresponding carry-free model with $h_{11}(z)=z^{3}+1, h_{12}(z)=$ $z+1, h_{21}(z)=z+1, h_{22}(z)=1$ has 2 d.o.f., since its determinant in the polynomial ring is non-zero. But

$$
Y=2^{k}\left[\begin{array}{ll}
5 & 2 \\
2 & 1
\end{array}\right] X+N,
$$

has 2 d.o.f. under the real Gaussian model. Under the carryfree model, the channel coefficients are $h_{11}(z)=z^{2}+$ $1, h_{12}(z)=z+1, h_{21}(z)=z+1, h_{22}(z)=1$, which lead to determinant 0 .
This inconsistency between the carry-free model and the Gaussian model seems to arise from the fact that numbers behave differently under the two algebras because of the lack of carryovers. For instance, 5, a prime integer, is composite in the carry-free ring $\left(101=11 \otimes_{c} 11\right)$. This is the primary difficulty in building a direct map from Gaussian instances to their carry-free counterparts.

\section{CHANNELS WITH UNKNOWN I.I.D. FADING}

\section{A. Point-to-point channel with unknown i.i.d. fading}

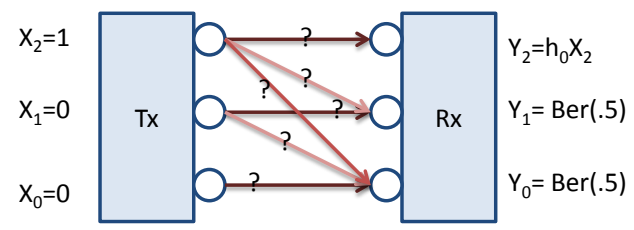

Fig. 4. This figure shows a carry-free model for an unknown fading channel. The maximal non-zero bit of the input contaminates output at all lower-levels. $y_{2}=h_{0} x_{2}$ remains correlated to $x(z)$. However, $y_{1}=h_{0} x_{1}+h_{-1} x_{2}$, is now a Bernoulli- $-\frac{1}{2}$ random variable independent of $x(z)$ if $x_{2}=1$. The same is true for all lower levels of $y(z)$.

1) Point-to-point carry-free model: Consider the carry-free model for a channel with unknown i.i.d fading as in Fig. 4. The basic setup builds from earlier sections ${ }^{3}: y(z)=\lfloor h(z) x(z)\rfloor$ where $h(z)=h_{0}+h_{-1} z^{-1}+\cdots$ and $x(z)=x_{n} z^{n}+$ $x_{n-1} z^{n-1}+\cdots+x_{0}$. Here, $h_{i}$ are i.i.d. Bernoulli- $\frac{1}{2}$ random variables which are unknown to both the transmitter and the receiver, and $x_{i} \in\{0,1\}$.

The first thing to note is that this carry-free channel is not deterministic - the randomness from the fading introduces randomness in the input-output relationship. Next, observe that once the maximum bit level in the output is fixed, the randomness introduced by the channel forces all bits below the maximum level to be Bernoulli- $\frac{1}{2}$ random variables which are independent from the input. Therefore, we focus our attention on the highest 1 received in $y(z)$. Define,

$$
y_{q}= \begin{cases}-1 & \text { if } y(z)=0 \\ \max \left\{i \mid y_{i}=1\right\} & \text { if } y(z) \neq 0\end{cases}
$$

$x_{q}$ is similarly defined.

The key observation is that $x_{q}$ and $y_{q}$ are sufficient statistics of $x(z), y(z)$ for $y(z), x(z)$ respectively, and this is captured in the following lemma.

Lemma 5.1: $P\left(y_{q}=x_{q}-i\right)=\left(\frac{1}{2}\right)^{i+1}$ for $0 \leq i \leq x_{q}$ and $P\left(y_{q}=-1\right)=\left(\frac{1}{2}\right)^{x_{q}+1}$. Moreover, $\quad x(z)-x_{q}-y_{q}-y(z)$ form a Markov chain, and $I(y(z) ; x(z))=I\left(y_{q} ; x(z)\right)=$ $I\left(y_{q} ; x_{q}\right)$.

The proof of this lemma is simple and omitted for space constraints. We use the lemma to calculate the capacity of this channel, $C_{c f}=\max _{p(x(z))} I(x(z) ; y(z))$.

Theorem 5.2: The capacity of the carry-free point-to-point channel is $\log (n+2)-3 \leq C_{c f} \leq \log (n+2)$.

\footnotetext{
${ }^{3}$ For ease of exposition we adopt slightly different model parameters and incorporate the channel power gain $z^{n+1}$ (not $z^{n}$ ) into $x(z)$ and $h(z)$.
} 
The achievable scheme involves using monomials $z^{x_{q}}$ as the channel input $x(z)$ and parallels pulse-position modulation. The converse follows from Lemma 5.1.

2) Point-to-point max-model: The observations above suggest that the essence of communicating over unknown fading channels is captured by the highest non-zero bits of the input and output. Hence, we propose the max-model, which depends only on the maximal bit of the signal. Let $x \in\{0,1,2 \cdots n\}$ represent the position of the maximal bit in the input signal. Then, $y$, the position of the maximal bit in the output signal is represented as simply as

$$
y=x .
$$

It is easy to see that the capacity of this channel is $C_{\max }=$ $\log (n+1)$.

3) Point-to-point real model: The key point of this section is to give an intuitive explanation of the $\log \log S N R$ result for communication over unknown fading channels. For a channel with uniform distribution: $Y=H \cdot X+W$, unknown i.i.d. fading $H \sim U[0,1]$, input power constraint $\mathbb{E}\left[X^{2}\right] \leq P$ and noise $W \sim U[0,1]$.

The optimal achievable scheme for this channel is to modulate the energy-level, and there are $n=\log P$ of those available. The different signal values (logarithmically spaced) which are used as inputs can be thought of as pulse-positions for standard pulse-position-modulation. The observation that $\lfloor\log |Y|\rfloor^{+}$is an approximate sufficient statistic for $Y$, clearly illustrates the insight and applicability of the max-model to this problem.

\section{B. Multiple-access channel with unknown i.i.d fading}

1) The carry-free MAC: Next we move to the carryfree unknown MAC. $y(z)=\left\lfloor h(z) a(z)+h^{\prime}(z) b(z)\right\rfloor$ where $h(z)=h_{0}+h_{-1} z^{-1}+\cdots$ and $h^{\prime}(z)=h_{0}^{\prime}+h_{-1}^{\prime} z^{-1}+\cdots$ are unknown at the transmitter and receiver. Let $a(z)=a_{n_{1}} z^{n_{1}}+\cdots+a_{0} \quad$ and $\quad b(z)=b_{n_{2}} z^{n_{2}}+\cdots+b_{0}$, and $y_{q}, a_{q}$, and $b_{q}$ are defined as in the previous section.

Just as in the point-to-point case, the randomness introduced by the fading restricts the information communicated across the channel to the maximal power level over the two input signal, and $y_{q}$ depends only on $\max \left\{a_{q}, b_{q}\right\}$. This intuition is captured in Lemma 5.3. We omit the proof.

Lemma 5.3: $P\left(y_{q}=\max \left\{a_{q}, b_{q}\right\}-i\right)=\left(\frac{1}{2}\right)^{i+1} \quad$ for $0 \leq i \leq \max \left\{a_{q}, b_{q}\right\}$ and $P\left(y_{q}=-1\right)=\left(\frac{1}{2}\right)^{\max \left\{a_{q}, b_{q}\right\}+1}$. Further, $\quad a(z), b(z)-a_{q}, b_{q}-\max \left\{a_{q}, b_{q}\right\}-y_{q}-y(z)$ form a Markov chain. Hence, $I(y(z) ; a(z), b(z))=I\left(y_{q} ; a(z), b(z)\right)=I\left(y_{q} ; a_{q}, b_{q}\right)$.

Just as in the point-to-point case, Lemma 5.3 suggests a deterministic model, described in the next section.

2) The max-superposition MAC: Finally, we come to the example which gives the max-superposition model its name. Let $a, b \in\{0,1,2 \cdots n\}$ represent the highest non-zero bits of the two input signals in a MAC. Then the output, $y$, of the channel depends only on the maximum of the two signals:

$$
y=\max \{a, b\} .
$$

The very nature of this model suggests that it is not possible for both encoders to simultaneously communicate information. Theorem 5.4 below proves that the timesharing rate region is optimal to within one bit.

Theorem 5.4: The achievable rate region for the maxsuperposition MAC consists of all positive rate pairs $\left(R_{1}, R_{2}\right)$ such that

$$
\log \left(n_{2}+1\right) R_{1}+\log \left(n_{1}+1\right) R_{2} \leq \log \left(n_{1}+1\right) \log \left(n_{2}+1\right)
$$

Further, this region is tight to within 1 bit for both $R_{1}$ and $R_{2}$ - namely all achievable pairs must satisfy:

$$
\begin{array}{r}
\log \left(n_{2}+1\right)\left(R_{1}-1\right)+\log \left(n_{1}+1\right)\left(R_{2}-1\right) \leq \\
\log \left(n_{1}+1\right) \log \left(n_{2}+1\right)
\end{array}
$$

3) The real MAC: Consider the real valued MAC: $Y=H \cdot A+H^{\prime} \cdot B+W$, with $H, H^{\prime}$ and $W$ are independent $U[0,1]$ and unknown at the transmitter and receiver, and $A, B$ are subject to quadratic power constraints $P_{1}$ and $P_{2}$. The maximum bit-levels $n_{1}, n_{2}$ from the carry free model are equal to $n_{1}=\log P_{1}, n_{2}=\log P_{2}$. Like the max-superposition MAC, we can prove a theorem similar to Thm. 5.4 and prove that timesharing is approximately optimal.

\section{Relay network}

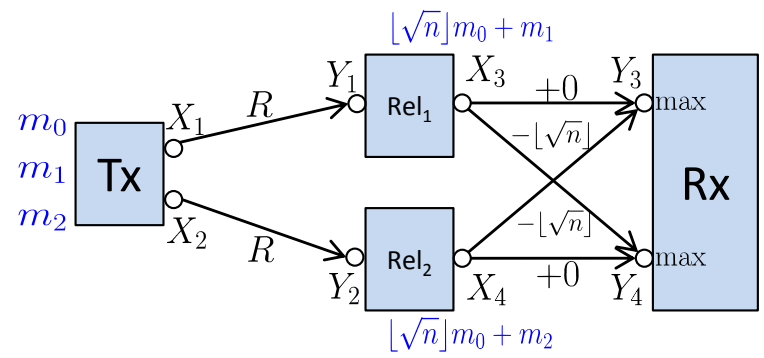

Fig. 5. A max-superposition relay network with an approximately optimal partial-decode-and-forward scheme. The cross-links between $X_{3}, X_{4}$ and $Y_{3}, Y_{4}$ are weaker than the direct links and lead to downward bit-level shifts by $\lfloor\sqrt{n}\rfloor . X_{1}=\lfloor\sqrt{n}\rfloor m_{0}+m_{1}$ and $X_{2}=\lfloor\sqrt{n}\rfloor m_{0}+m_{2}$ are transmitted by the two relays.

At first glance, it would seem that there is no point to even thinking about coordination or beam-forming style gains by the relays in an unknown fading network. Compress-andforward seems like the right approximately optimal scheme. However, this section gives an example of a relay network where surprisingly, partial-decode-and-forward can infinitely outperform compress-and-forward style schemes, and there is a significant coordination gain-traditionally known as beamforming gain - to be exploited. This observation is quite interesting when we remind ourselves that the beam-forming gain is usually bounded by a constant in known-fading Gaussian networks with linear superposition, and compress-and-forward is the optimal in linear ADT relay networks [2]. ${ }^{4}$

\footnotetext{
${ }^{4}$ It was already known that partial-decode-and-forward is also optimal in semi-deterministic single-relay channels [11].
} 
In the previous section, we saw that the max operation essentially captures superposition in i.i.d. unknown fading networks. We focus on a specific max-superposition relay network shown in Fig. 5. Channels connecting $X_{1}$ to $Y_{1}$ and $X_{2}$ to $Y_{2}$ are orthogonal noiseless links with rate $R$ each. The MIMO channel connecting $X_{3}, X_{4}$ to $Y_{3}, Y_{4}$ is a max-superposition deterministic model. We consider $X_{3} \in$ $\{0,1, \cdots, n\}, X_{4} \in\{0,1, \cdots, n\}$ and

$$
\begin{aligned}
& Y_{3}=\max \left(X_{3},\left(X_{4}-\lfloor\sqrt{n}\rfloor\right)^{+}\right) \\
& Y_{4}=\max \left(\left(X_{3}-\lfloor\sqrt{n}\rfloor\right)^{+}, X_{4}\right)
\end{aligned}
$$

The shifted max-operation captures the difference between the direct-link and cross-link gains in the original Gaussian MIMO channel. The cross links are weaker than the direct links, but not by much in a proper scaling.

First, observe that when $X_{3}-X_{4} \geq \sqrt{n}, Y_{3}=X_{3}$ and $Y_{4}=\left(X_{3}-\lfloor\sqrt{n}\rfloor\right)^{+}$. Thus, both the received signals only depend on $X_{3}$, and $X_{4}$ is useless. Moreover, when $X_{3}$ and $X_{4}$ are independent and their ranges are large, with high probability the difference between $X_{3}$ and $X_{4}$ is greater than $\sqrt{n}$ and both $Y_{3}$ and $Y_{4}$ only depend on either $X_{3}$ or $X_{4}$. Therefore, in compress-and-forward (where $X_{3}$ and $X_{4}$ are chosen independently), the throughput is essentially bounded by $\log n$ which is the maximum entropy of a single input.

However, decode-and-forward can exploit the coordination gain between $X_{3}$ and $X_{4}$. We can keep the difference within $\sqrt{n}$ and let $Y_{3}, Y_{4}$ depend on both $X_{3}, X_{4}$. The key observation is that when the quotients of $X_{3}, X_{4}$ divided by $\lfloor\sqrt{n}\rfloor$ are the same, $X_{3}, X_{4}$ have to be within $\sqrt{n}$. To keep the quotients the same, we transfer a common message $m_{0}$ to both relays as the common quotient.

An approximately optimal partial-decode-and-forward scheme is described in Fig. 5. The transmitter divides the message into three parts $m_{0}, m_{1}, m_{2} \in\{0,1, \cdots,\lfloor\sqrt{n}\rfloor-1\}$. The common message $m_{0}$ is sent to both relays and used as a quotient. The private messages $m_{1}$ and $m_{2}$ are transmitted to $R e l_{1}$ and $R e l_{2}$ respectively and used as remainders. In other words, $X_{3}$ and $X_{4}$ are chosen as $\lfloor\sqrt{n}\rfloor m_{0}+m_{1}$ and $\lfloor\sqrt{n}\rfloor m_{0}+m_{2}$ respectively. Since the signals are within $\sqrt{n}$, $Y_{3}$ and $Y_{4}$ also have to equal $X_{3}$ and $X_{4}$ (eqs. (9), (10)). Moreover, since the ranges of $m_{0}, m_{1}, m_{2}$ are $\lfloor\sqrt{n}\rfloor$, the achievable rate of this partial-decode-and-forward can increase to $\frac{3}{2} \log n$.

We summarize our understanding in Thm. 5.5 and Fig. 6. The real-channel counterparts are not hard to derive.

Theorem 5.5: Let $C_{\text {rel }}$ be the capacity of the relay channel given as above. The cut-set bound gives the upperbound

$C_{r e l} \leq \min \left\{2 R, R+\frac{1}{2} \log (n+1)+2 \log 3, \frac{3}{2} \log (n+1)+\frac{3}{2} \log 2\right\}$

The proposed partial-decode-and-forward scheme gives the following lower bound.

$$
C_{r e l} \geq \min \{2 R, R+\log (\lfloor\sqrt{n}\rfloor), 3 \log (\lfloor\sqrt{n}\rfloor)\}
$$

This scheme is approximately optimal within a constant gap. Since compress-and-forward cannot exploit a "beam-forming gain", it can perform infinitely worse than the partial-decodeand-forward scheme.

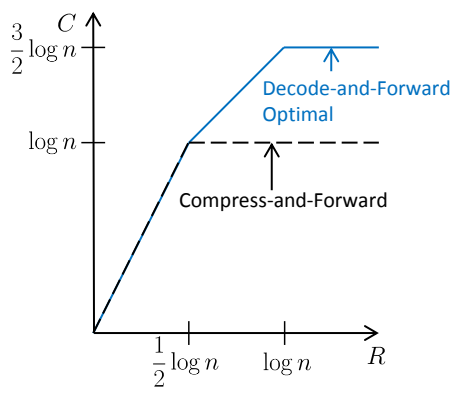

Fig. 6. Generalized d.o.f. plot for the relay network in Fig. 5. $\frac{3}{2}$ d.o.f. capacity is only achievable by partial-decode-and-forward, not by compressand-forward. Therefore, the gap between the two goes to infinity as $n$ grows.

\section{CONCLUSION}

In this paper, we explore carry-free models and their various applications. The ability of carry-free models to capture diverse channel gains illustrates how real-interference alignment works in Gaussian models. This diversity also allows us to model unknown fading. We also observe that in unknown fading networks the carry-free models can be further simplified to max-superposition models. These max-superposition networks show a curious property-arbitrarily large "beamforming" like gains-that needs more exploration.

\section{ACKNOWLEDGMENTS}

The authors would like to thank the NSF for grants NSF07291222, NSF-0917212, and NSF-0932410, Guy Bresler and Abolfazl Motahari for helpful discussions, and the reviewers for suggested changes to the results.

\section{REFERENCES}

[1] U. Niesen and M. Maddah-Ali, "Interference alignment: From degreesof-freedom to constant-gap capacity approximations," Arxiv preprint arXiv:1112.4879, 2011.

[2] A. Avestimehr, S. Diggavi, and D. Tse, "Wireless network information flow: A deterministic approach," Information Theory, IEEE Transactions on, vol. 57, no. 4, pp. 1872-1905, 2011.

[3] M. Anand and P. Kumar, "A digital interface for Gaussian relay and interference networks: Lifting codes from the discrete superposition model," Arxiv preprint arXiv:1005.0167, 2010.

[4] G. Bresler and D. Tse, "The two-user Gaussian interference channel: a deterministic view," European Transactions on Telecommunications, vol. 19, no. 4, pp. 333-354, 2008.

[5] J. Richters, "Communication over fading dispersive channels," DTIC Document, Tech. Rep., 1967.

[6] G. Taricco and M. Elia, "Capacity of fading channel with no side information," Electronics Letters, vol. 33, no. 16, pp. 1368-1370, 1997.

[7] Abou-Faycal, I.C. and Trott, M.D. and Shamai, S., "The capacity of discrete-time memoryless Rayleigh-fading channels," Information Theory, IEEE Transactions on, vol. 47, no. 4, pp. 1290-1301, 2001.

[8] A. Lapidoth and S. Moser, "Capacity bounds via duality with applications to multiple-antenna systems on flat-fading channels," Information Theory, IEEE Transactions on, vol. 49, no. 10, pp. 2426-2467, 2003.

[9] A. Motahari, S. Gharan, and A. Khandani, "Real interference alignment with real numbers," Arxiv preprint arxiv:0908.1208, 2009.

[10] Y. Wu, S. Shamai, and S. Verdú, "Degrees of freedom of the interference channel: A general formula," in Information Theory Proceedings (ISIT), 2011 IEEE International Symposium on. IEEE, 2011, pp. 1362-1366.

[11] Y.-H. Kim, "Capacity of a class of deterministic relay channels," Information Theory, IEEE Transactions on, vol. 54, no. 3, pp. 1328 -1329 , march 2008. 